Original Research Paper

\title{
Derivation of Potentially Important Masses for Physics and Astrophysics by Dimensional Analysis
}

\author{
Dimitar Valev \\ Space Research and Technology Institute, \\ Bulgarian Academy of Sciences, 6000 Stara Zagora, Bulgaria
}

Email: valev@gbg.bg

\begin{abstract}
The Hubble constant has been added in addition to the three fundamental constants (speed of light, gravitational constant and Planck constant) used by Max Planck, for derivation of the Planck mass by dimensional analysis. As a result, a general solution is found for the mass dimension expression $m=\gamma^{p} \mathrm{~m}_{0}$, where $m_{0} \equiv m_{p}$ is the Planck mass, $\gamma=$ $1.23 \times 10^{-61}$ is a small dimensionless quantity and $p$ is an arbitrary parameter in the interval $[-1,1]$. The Planck mass $m_{p}=2.17 \times 10^{-8} \mathrm{~kg}$, mass of the Hubble sphere $M_{H} \sim 10^{53} \mathrm{~kg}$, minimum quantum of mass $/$ energy $m_{G}=2.68 \times 10^{-69} \mathrm{~kg}$, Weinberg mass $m_{W}=1.08 \times 10^{-28} \mathrm{~kg}$, mass of hypothetical quantum gravity atom $M_{G}=3.8 \times 10^{12} \mathrm{~kg}$, Eddington mass limit of stars $M_{E}=6.6 \times 10^{32} \mathrm{~kg}$ and some more masses potentially important for the physics and astrophysics represent particular solutions for values of $p$, expressed as fractions with small numerators and nominators.
\end{abstract}

Keywords: Dimensional Analysis, Fundamental Constants, Ockham's Razor, Mass of the Hubble Sphere, Weinberg Mass, Eddington Mass Limit

\section{Introduction}

The dimensional analysis is a conceptual tool often applied in physics and astrophysics to understand physical situations involving certain physical quantities (Bridgman, 1922; Kurth, 1972; Bhaskar and Nigam, 1990; Petty, 2001). It is routinely used to check the plausibility of the derived equations and computations. When it is known with which other determinative quantities a particular quantity would be connected, but the form of this connection is unknown, a dimensional equation $\left[q_{0}\right] \sim \prod_{i=1}^{n}\left[q_{i}\right]^{n_{i}}$ is composed for its finding. In the left side of the equation is placed the unit of this quantity $q_{0}$ with its dimensional exponent and in the right side of the equation is placed the product of units of the determinative quantities $q_{i}$ raised to the unknown exponents $n_{i}$, where $\mathrm{n}$ is a positive integer and the exponents $n_{i}$ are rational numbers. Most often, the dimensional analysis is applied in the mechanics, aerodynamics, astrophysics and other fields of the modern physics, where there are many problems with a few determinative quantities.

The Planck mass as defined by Planck (1906) in terms of three fundamental constants, speed of light in vacuum (c), gravitational constant $(\mathrm{G})$ and reduced Planck constant $\hbar$, is $m_{P}=\sqrt{\frac{\hbar c}{G}}$. Since the constants $c$, $G$ and $\hbar$ represent three very basic aspects of the universe (i.e. the relativistic, gravitational and quantum phenomena), the Planck mass appears to a certain degree a unification of these phenomena. The Planck mass has many important theoretical ramifications in modern physics. One of them is that the energy equivalent of Planck mass $E_{P}=m_{P} c^{2}=\sqrt{\frac{\hbar c^{5}}{G}} \sim 10^{19} \mathrm{GeV}$ appears to be the unification energy of the fundamental interactions (Georgi et al., 1974). Additionally, the Planck mass can be derived approximately by defining it as a mass whose Compton wavelength and Schwartzchild radius are equal (Bergmann, 1992).

The Hubble constant $H$ has been added to the set of constants $c, G, \hbar$ and thus a unique mass dimension quantity has been derived for every triad $(c, G, H),(c, \hbar$, $H)$ and $(G, \hbar, H)$ by dimensional analysis (Valev, 2013). Thus, three new fundamental masses are found, i.e. $m_{1}=\frac{c^{3}}{G H} \sim 10^{53} \mathrm{~kg}, \quad m_{2}=\frac{\hbar H}{c^{2}}=2.68 \times 10^{-69} \mathrm{~kg} \quad$ and
$m_{3}=\sqrt[5]{\frac{H \hbar^{3}}{G^{2}}}=1.43 \times 10^{-20} \mathrm{~kg}$. The mass $m_{1}$ is identified 
with the mass of the Hubble sphere $M_{H}=\frac{c^{3}}{2 G H}=8.8 \times 10^{52} \mathrm{~kg}, m_{2}$ with minimum quantum of mass/energy and $m_{3}$ is conjectured to be the mass of a yet unknown super heavy particle or fundamental energetic scale. According to the contemporary cosmology, the Hubble constant slowly decreases with the age of the universe $\dot{H} / H \sim-H=-2.3 \times 10^{-18} \mathrm{~s}^{-1}$, i.e. $H$ decreased only $33 \%$ from the Solar system formation till now.

In the present work we seek a mass dimension quantity that represents as a product of rational exponents of the four constants $-c, G, \hbar$ and $H$.

\section{General Solution of the Problem for Finding of a Mass Dimension Quantity by Means of Fundamental Constants $c, G$ and $H$}

By dimensional analysis, we search for a mass dimension quantity $\mathrm{m}$ in the form of product of rational exponents $n_{1}, n_{2}, n_{3}$ and $n_{4}$ of the constants $c, G, \hbar$ and $H$ :

$$
m=k c^{n_{1}} G^{n_{2}} \hbar^{n_{3}} H^{n_{4}} \sim c^{n_{1}} G^{n_{2}} \hbar^{n_{3}} H^{n_{4}}
$$

The exponents $n_{1}, n_{2}, n_{3}$ and $n_{4}$ are unknown quantities that can be found by matching dimensions on both sides of equation (1) and $\mathrm{k}$ is a dimensionless parameter (coefficient) on the order of unity.

Replacing dimensions of $m, c, G, \hbar$ and $H$ in (1) we find the dimensional equation:

$$
L^{0} T^{0} M^{1}=L^{n_{1}+3 n_{2}+2 n_{3}} T^{-n_{1}-2 n_{2}-n_{3}-n 4} M^{-n_{2}+n_{3}}
$$

From Equation (2) we find system of linear equations for unknown quantities $n_{1}, n_{2}, n_{3}$ and $n_{4}$ :

$$
\begin{aligned}
& n_{1}+3 n_{2}+2 n_{3}=0 \\
& -n_{1}-2 n_{2}-n_{3}-n_{4}=0 \\
& -n_{2}+n_{3}=1
\end{aligned}
$$

The rank of augmented matrix of the system $r=3$ is equal to the rank of the coefficient matrix. Thus, in accordance with the Rouche-Capelli theorem the system is consistent and so must have at least one solution. The solution is unique if and only if the rank equals the number of variables. In the system (3) the number of variables $m=4>r=3$, therefore the solution is not unique, but having infinitely many solutions. However, upon introducing the concept of a free parameter $p$, where in it is accepted that $n_{4}=p$, system (3) can be transform to:

$$
\begin{aligned}
& n_{1}+3 n_{2}+2 n_{3}=0 \\
& -n_{1}-2 n_{2}-n_{3}=p \\
& -n_{2}+n_{3}=1
\end{aligned}
$$

The determinant of system (4) is $\Delta=2 \neq 0$ and the system has a solution that is dependent upon a free parameter $p$. We find the solution of the system (4) by means of Cramer's rule:

$$
\begin{aligned}
& n_{1}=(1-5 p) / 2, \\
& n_{2}=(p-1) / 2, \\
& n_{3}=(p+1) / 2, \\
& n_{4}=p
\end{aligned}
$$

where, $p$ is a free parameter.

Replacing the solution (5) in Equation (1) we find Equation (6) for the mass $m$ :

$$
m \sim c^{(1-5 p) / 2} G^{(p-1) / 2} \hbar^{(p+1) / 2} H^{p}
$$

Obviously, the Equation (6) can be transformed in Equation (7):

$m \sim c^{\frac{1}{2}-\frac{5}{2} p} G^{-\frac{1}{2}+\frac{p}{2}} \hbar^{\frac{1}{2}+\frac{p}{2}} H^{p}=\sqrt{\frac{c \hbar}{G}}\left(\sqrt{\frac{G \hbar H^{2}}{c^{5}}}\right)^{p}$

Therefore, we find the general solution (8):

$$
m \sim \gamma^{p} m_{0}
$$

where, $\quad \gamma=\sqrt{\frac{G \hbar H^{2}}{c^{5}}} \sim 10^{-61} \quad$ is exceptionally small dimensionless quantity, $m_{0} \equiv m_{P}=\sqrt{\frac{\hbar c}{G}}=2.17 \times 10^{-8} \mathrm{~kg}$ is the Planck mass and $p$ is a free parameter.

Although the parameter $p$ can take arbitrary values in the interval $(-\infty,+\infty)$, only solutions in the interval $[-1$, 1] could have physical meaning, because for limit values $p=-1$ and $p=1$ the resulting solutions are, respectively, the mass of the Hubble sphere $M_{H} \sim m_{1}=\frac{c^{3}}{G H}=1.76 \times 10^{53} \mathrm{~kg}$ (Carvalho, 1995; Valev, 2009) and the minimum measurable mass/energy in the universe $m_{2} \equiv m_{G}=\frac{\hbar H}{c^{2}}=2.68 \times 10^{-69} \mathrm{~kg}=1.5 \times 10^{-33} \mathrm{eV}$ (Sivaram, 1982; Alfonso-Faus, 2012). The exceptionally small mass $m_{3}$ seems close to the graviton mass obtained by different methods (Woodward et al., 1975; Gershtein et al., 1998; Valev, 2008; Alves et al., 2011).

According to Ockham's razor principle, all other things being equal, the simplest theory is the most likely to be true (Rodriguez-Fernandez, 1999). In science, this principle is used as a heuristic technique (discovery tool) to guide scientists in the development of theoretical models (Gauch, 2003). Therefore, in the following section, we consider particular solutions where the free parameter $|p| \leq 1$ represents as a fraction with a small numerator and denominator, 
i.e. $p=0, \pm 1, \pm \frac{1}{3}, \pm \frac{1}{2}, \pm \frac{1}{4}, \pm \frac{1}{5}, \pm \frac{2}{3}$. We will show that some such solutions result in mass formulas that could be interesting for contemporary particle physics and astrophysics.

\section{Particular Solutions where the Free Parameter $|\mathbf{p}| \leq 1$ Represents as Fractions having Small Numerators and Denominators}

From the general solution (8) we find the Planck mass $m_{\mathrm{p}}$ as a particular solution (9) in case of $p=0$ :

$$
m_{0} \equiv m_{P}=\sqrt{\frac{\hbar c}{G}}=2.17 \times 10^{-8} \mathrm{~kg}
$$

As it has been mentioned in Section 2, from Equation (8) we find mass of the Hubble sphere $M_{H}$ as a particular solution (10) at $p=-1$ :

$$
M_{H} \sim m_{1}=\frac{c^{3}}{G H}=1.76 \times 10^{53} \mathrm{~kg}
$$

Analogously, from the general solution (8) we find the minimum quantum of mass/energy $m_{G}$ as a particular solution (11) at $p=1$ :

$$
\begin{aligned}
m_{2} & \equiv m_{G}=\frac{\hbar H}{c^{2}} \\
& =2.68 \times 10^{-69} \mathrm{~kg}=1.5 \times 10^{-33} \mathrm{eV}
\end{aligned}
$$

From Equation (8) we find the particular solution (12) at $p=\frac{1}{3}$ :

$$
\begin{aligned}
m_{4} & \equiv m_{W}=c^{-\frac{1}{3}} G^{-\frac{1}{3}} \hbar^{\frac{2}{3}} H^{\frac{1}{3}}=\sqrt[3]{\frac{H \hbar^{2}}{c G}} \\
& =1.08 \times 10^{-28} \mathrm{~kg}=60.8 \mathrm{MeV}
\end{aligned}
$$

The Equation (12) represents the well known Weinberg mass formula (Weinberg, 1972). The physical meaning of the Weinberg mass was found from Sivaram (1982). He shows the Weinberg mass represents the lightest mass whose self-gravitational energy has measurable value for the time of existence of the universe $H^{1} \approx 1.38 \times 10^{10}$ years.

From the general solution (8) we find the particular solution (13) at $p=-\frac{1}{3}$ :

$$
m_{5}=c^{\frac{4}{3}} G^{-\frac{2}{3}} \hbar^{\frac{1}{3}} H^{-\frac{1}{3}}=c \cdot \sqrt[3]{\frac{c \hbar}{G^{2} H}}=4.36 \times 10^{12} \mathrm{~kg}
$$

It has been shown (Forsythe, 2009) that the hypothetical 'Quantum gravity atom' built up from a central electro-neutral mass $M_{G}$ around which orbits an electro-neutral particle of electron mass $m_{e}$ at a distance equal to the Bohr radiu $a_{0}=\frac{\hbar^{2}}{m_{e} e^{2}}=5.3 \times 10^{-11} \mathrm{~m}$, possesses gravitational potential $V=\frac{G M_{G} m_{e}}{a_{0}}$ equal to the electrostatic potential $V_{E}=\frac{e^{2}}{a_{0}}$. From here Forsythe finds the central mass $M_{G}$ :

$M_{G}=\frac{e^{2}}{G m_{e}}=3.8 \times 10^{12} \mathrm{~kg} \sim m_{5}$

From the general solution (8) we find the particular solution (15) at $p=\frac{1}{2}-$ :

$$
\begin{aligned}
m_{6} & =c^{-\frac{3}{4}} G^{-\frac{1}{4}} \hbar^{\frac{3}{4}} H^{\frac{1}{2}}=\sqrt[4]{\frac{\hbar^{3} H^{2}}{G c^{3}}} \\
& =7.64 \times 10^{-39} \mathrm{~kg}=4.3 \times 10^{-3} \mathrm{eV}
\end{aligned}
$$

Obviously, the mass $m_{6}$, obtained from Equation (8) at $p=\frac{1}{2}$ is of the order of the neutrino rest mass (Goobar et al., 2006).

From (8) we find the particular solution (16) at $p=-\frac{1}{2}$ :

$$
m_{7}=c^{\frac{7}{4}} G^{-\frac{3}{4}} \hbar^{\frac{1}{4}} H^{-\frac{1}{2}}=c \cdot \sqrt[4]{\frac{c^{3} \hbar}{G^{3} H^{2}}}=6.18 \times 10^{22} \mathrm{~kg}
$$

The mass $m_{1}$ represents approximately $1 \%$ of the Earth mass $M_{\text {Earth }}=5.97 \times 10^{24} \mathrm{~kg}$ and is close to the Moon mass $M_{\text {Moon }}=7.34 \times 10^{22} \mathrm{~kg}$, that presents typical satellite in the Solar system.

From the general solution (8) we find the particular solution (17) at $p=\frac{1}{4}$ :

$$
\begin{aligned}
m_{8} & =c^{-\frac{1}{8}} G^{-\frac{3}{8}} \hbar^{\frac{5}{8}} H^{\frac{1}{4}}=\sqrt[8]{\frac{\hbar^{5} H^{2}}{c G^{3}}} \\
& =1.29 \times 10^{-23} \mathrm{~kg}=7.25 \mathrm{TeV}
\end{aligned}
$$

This energy is typical for the energy of protons in Large Hadron Collider ( $L H C$ ) and possibly is connected with mass of yet unobserved heavy particle or fundamental energetic scale.

From (8) we find the particular solution (18) at $p=-\frac{1}{4}$ :

$$
m_{9}=c^{\frac{9}{8}} G^{-\frac{5}{8}} \hbar^{\frac{3}{8}} H^{-\frac{1}{4}}=c \cdot \sqrt[8]{\frac{c \hbar^{3}}{G^{5} H^{2}}}=3.67 \times 10^{7} \mathrm{~kg}
$$


The mass $m_{9}$ most probably has no reference to the fundamental physics.

From the general solution (8) we find the particular solution (19) at $p=-\frac{1}{5}$ :

$$
m_{10}=c G^{-\frac{3}{5}} \frac{2}{\hbar^{\frac{1}{5}}} H^{-\frac{1}{5}}=c \cdot \sqrt[5]{\frac{\hbar^{2}}{G^{3} H}}=3.4 \times 10^{4} \mathrm{~kg}
$$

The mass $m_{10}$ hardly have some physical meaning.

The case $p=\frac{1}{5}$ uniquely yields the mass equation (20):

$$
m_{3}=\sqrt[5]{\frac{H \hbar^{3}}{G^{2}}}=1.43 \times 10^{-20} \mathrm{~kg}=8.0 \times 10^{6} \mathrm{GeV}
$$

This mass also can't be identified but possibly could be considered a heuristic prediction of the suggested model for a super heavy unobserved particle or

fundamental energetic scale intermediate for electroweak scale $\sim 250 \mathrm{GeV}$ and $\mathrm{GUT}$ scale $\sim 10^{16} \mathrm{GeV}$.

From (8) we find the particular solution (21) at $p=\frac{2}{3}$ :

$$
\begin{aligned}
m_{11} & =c^{-\frac{7}{6}} G^{-\frac{1}{6}} \hbar^{\frac{5}{6}} H^{\frac{2}{3}}=\frac{1}{c} \cdot \sqrt[6]{\frac{\hbar^{5} H^{4}}{c G}} \\
& =5.39 \times 10^{-49} \mathrm{~kg}=3.0 \times 10^{-13} \mathrm{eV}
\end{aligned}
$$

This mass is close to one of the seven fundamental equidistant masses found in (Forsythe and Valev, 2014), namely the mass $M_{(-1)}=7.15 \times 10^{-49} \mathrm{~kg}$.

Finally, from the general solution (8) we find the particular solution (22) at $p=-\frac{2}{3}$ :

$$
\begin{aligned}
m_{12} & =c^{\frac{13}{6}} G^{-\frac{5}{6}} \hbar^{\frac{1}{6}} H^{-\frac{2}{3}}=c^{2} \cdot \sqrt[6]{\frac{c \hbar}{G^{5} H^{4}}} \\
& =8.76 \times 10^{32} \mathrm{~kg}
\end{aligned}
$$

\begin{tabular}{|c|c|c|}
\hline$p$ & Mass corresponding to $p$ & Identification of mass $m_{i}$ \\
\hline-1 & $m_{1}=\frac{c^{3}}{G H}=1.76 \times 10^{53} \mathrm{~kg}$ & Mass of the Hubble sphere $M_{H}$ \\
\hline$-\frac{2}{3}$ & $m_{12}=c^{2} \cdot \sqrt[6]{\frac{c \hbar}{G^{5} H^{4}}}=8.76 \times 10^{32} \mathrm{~kg}$ & Eddington mass limit of stars $M_{E}$ \\
\hline$-\frac{1}{2}$ & $m_{7}=c \cdot \sqrt[4]{\frac{c^{3} \hbar}{G^{3} H^{2}}}=6.18 \times 10^{22} \mathrm{~kg}$ & Mass of the Moon (Typical satellite in the Solar system) \\
\hline$-\frac{1}{3}$ & $m_{5}=c \cdot \sqrt[3]{\frac{c \hbar}{G^{2} H}}=4.36 \times 10^{12} \mathrm{~kg}$ & Mass of 'Quantum Gravity Atom' $M_{G}$ \\
\hline$-\frac{1}{4}$ & $m_{9}=c \cdot \sqrt[8]{\frac{c \hbar^{3}}{G^{5} H^{2}}}=3.67 \times 10^{7} \mathrm{~kg}$ & - \\
\hline$-\frac{1}{5}$ & $m_{10}=c \cdot \sqrt[5]{\frac{\hbar^{2}}{G^{3} H}}=3.40 \times 10^{4} \mathrm{~kg}$ & - \\
\hline 0 & $m_{0}=\sqrt{\frac{c \hbar}{G}}=2.17 \times 10^{-8} \mathrm{~kg}$ & Planck mass $m_{p}$ \\
\hline$\frac{1}{5}$ & $m_{3}=\sqrt[5]{\frac{H \hbar^{3}}{G^{2}}}=1.4310^{-20} \mathrm{~kg}$ & Prediction for super massive unknown particle \\
\hline$\frac{1}{4}$ & $m_{8}=\sqrt[8]{\frac{\hbar^{5} H^{2}}{c G^{3}}}=1.29 \times 10^{-23} \mathrm{~kg}$ & Prediction for massive unknown particle \\
\hline$\frac{1}{3}$ & $m_{4}=\sqrt[3]{\frac{H \hbar^{2}}{c G}}=1.08 \times 10^{-28} \mathrm{~kg}$ & Weinberg mass $m_{W}$ \\
\hline$\frac{1}{2}$ & $m_{6}=\sqrt[4]{\frac{\hbar^{3} H^{2}}{G c^{3}}}=7.64 \times 10^{-39} \mathrm{~kg}$ & Neutrino mass $m_{v}$ \\
\hline$\frac{2}{3}$ & $m_{11}=\frac{1}{c} \cdot \sqrt[6]{\frac{\hbar^{5} H^{4}}{c G}}=5.39 \times 10^{-49} \mathrm{~kg}$ & Forsythe-Valev mass $M_{(-1)}$ \\
\hline 1 & $m_{2}=\frac{\hbar H}{c^{2}}=2.68 \times 10^{-69} \mathrm{~kg}$ & Minimum quantum of mass/energy (Graviton mass $m_{G}$ ) \\
\hline
\end{tabular}

Table 1. Masses whose free parameters are in the range $|p| \leq 1$ and appear in the general solution as fractions having small numerators and denominators 
The mass $m_{12}$ has been identified in (Forsythe and Valev, 2014) with Eddington mass limit of the most massive stars $M_{E}=6.6 \times 10^{32} \mathrm{~kg}$.

The above derived masses, whose free parameters are in the range $|p| \leq 1$ and appear in the general solution as fractions having small numerators and denominators, are presented in Table 1 .

Probably, the general solution (8) includes additional masses interesting from the physical view point, but indefiniteness of the parameter $p$ doesn't allow unambiguous finding of these masses.

Time dependence of some derived masses is natural and clear. For example mass of the Hubble sphere $M_{H}=\rho_{c} V_{H}=\frac{3 H^{2}}{8 \pi G} \frac{4 \pi}{3} \frac{c^{3}}{H^{3}}=\frac{c^{3}}{2 G H} \approx m_{1} \quad$ increase with cosmological expansion because of faster increase of volume (cubic) than decrease of total density $\bar{\rho}=\rho_{c}$ (quadratic). Same is valid for the minimum quantum of mass/energy $m_{2} \equiv m_{G}=\frac{\hbar H}{c^{2}} \sim 10^{-33} \mathrm{eV}$ and Weinberg mass $m_{4} \equiv m_{W}=\sqrt[3]{\frac{H \hbar^{2}}{c G}}=60.8 \mathrm{MeV}$ because they are related with Heisenberg's uncertainty principle $\Delta E \Delta t \geq \hbar$, where the duration of measurement is limited from the age of the universe $\Delta t \leq H^{-1}=1.38 \times 10^{10}=$ years (Sivaram, 1982). The Planck mass $m_{0} \equiv m_{P}=\sqrt{\frac{\hbar c}{G}}=2.17 \times 10^{-8} \mathrm{~kg}$ include constants c, G and $\hbar$ and is time independent, but the rest derived masses depend from the expansion. In result, the microscopic masses $m_{2}, m_{3}, m_{4}, m_{6}, m_{8}$ and $m_{11}$ decrease with cosmological expansion, while the macroscopic masses $m_{1}, m_{5}, m_{7}, m_{9}, m_{10}$ and $m_{12}$ and increase with the expansion.

\section{Conclusion}

The Hubble constant $H$ has been added to the three fundamental constants (the speed of light in vacuum, Newtonian gravitational constant and reduced Planck constant) used from Max Planck for derivation of Planck mass by dimensional analysis.

We search by dimensional analysis a mass dimension quantity that represents a product of rational exponents of the four constants $-c, G, \hbar$ and $H$. In result, a general solution has been found of mass dimension quantity $m=\gamma^{p} m_{0}$, where $m_{0} \equiv m_{P}=\sqrt{\frac{c \hbar}{G}}=2.17 \times 10^{-8} \mathrm{~kg} \quad$ is the Planck mass, $\quad \gamma=\sqrt{\frac{G \hbar H^{2}}{c^{5}}}=1.23 \times 10^{-61}$ is a small dimensionless quantity and $p$ is an arbitrary parameter in the interval $[-1,1]$. According to Ockham's razor principle, all other things being equal, the simplest theory is the most likely to be true. Therefore, we consider particular solutions where the free parameter $|p| \leq 1$ represents as a fraction with a small numerator and denominator, i.e. $p=0, \pm 1, \pm \frac{1}{3}, \pm \frac{1}{2}, \pm \frac{1}{4}, \pm \frac{1}{5}, \pm \frac{2}{3}$.

In result, it has been found that the Planck mass $m_{P}=\sqrt{\frac{c h}{G}}=2.17 \times 10^{-8} \mathrm{~kg}$, mass of the Hubble sphere $m_{H}=\frac{c^{3}}{G H} \sim 10^{53} \mathrm{~kg}$, minimum quantum of mass/energy $m_{G}=\frac{\hbar H}{c^{2}}=2.68 \times 10^{-69} \mathrm{~kg}, \quad$ Weinberg mass $m_{W}=\sqrt[3]{\frac{H \hbar^{2}}{c G}}=1.08 \times 10^{-28} \mathrm{~kg}, \quad$ mass of hypothetical quantum gravity atom $M_{G}=3.8 \times 10^{12} \mathrm{~kg}$, Eddington mass limit of stars $M_{E}=6.6 \times 10^{32} \mathrm{~kg}$ and some more masses potentially important for the physics and astrophysics represent particular solutions for values of $p$, expressed as fractions with small numerators and denominators. Likely, some of unidentified masses could have heuristic meaning for astrophysics and high energy physics.

\section{Acknowledgement}

I would like to thank Prof. Andrew Beckwith and Dr. Curtis Forsythe for encouraging discussions.

\section{Ethics}

This article is original and contains unpublished material. The corresponding author confirms that all of the other authors have read and approved the manuscript and no ethical issues involved.

\section{References}

Alfonso-Faus, A., 2012. Universality of the self gravitational potential energy of any fundamental particle. Astrophys. Space Sci., 337: 363-365.

DOI: $10.1007 / \mathrm{s} 10509-011-0803-\mathrm{x}$

Alves, M.E., O.D. Miranda and J.C. de Araujo, 2011. Can massive gravitons be an alternative to dark energy. Phys. Lett. B, 700: 283-288. DOI: $10.1016 /$ j.physletb.2011.05.022

Bergmann, P.G., 1992. The Riddle of Gravitation. Dover Publications, New York. ISBN: 0486273784

Bhaskar, R. and A. Nigam, 1990. Qualitative physics using dimensional analysis. Artificial Intelligence, 45: 73-111. DOI: 10.1016/0004-3702(90)90038-2

Bridgman, P.W., 1922. Dimensional Analysis. Yale Univ. Press, New Haven. ISBN: 0548910294

Carvalho, J.C., 1995. Derivation of the mass of the observable universe. Int. J. Theor. Phys. 34: 2507-2509. DOI: 10.1007/BF00670782 
Forsythe, C.J. and D.T. Valev, 2014. Extended mass relation for seven fundamental masses and new evidence of large numbers hypothesis. Phys. Int., 5: 152-158. DOI: $10.3844 /$ pisp.2014.152.158

Forsythe, C.J., 2009. Resonance structure of matter, nature of gravitation and the quantum energy states of the hydrogen atom. Phys. Essays, 22: 112-121. DOI: $10.4006 / 1.3100617$

Gauch, H.G., 2003. Scientific Method in Practice. Cambridge University Press, Cambridge, UK. ISBN: 0521017084

Georgi, H., H.R. Quinn and S. Weinberg, 1974. Hierarchy of interactions in unified gauge theories. Phys. Rev. Lett., 33: 451-454. DOI: 10.1103/PhysRevLett.33.451

Gershtein, S.S., A.A. Logunov and M.A. Mestvirishvili, 1998. On the upper limit for the graviton mass. Dokl. Phys., 43: 293-296

Goobar, A., S. Hannestad, E. Mortsell and H. Tu, 2006. The neutrino mass bound from WMAP 3 year data, the baryon acoustic peak, the SNLS supernovae and the Lyman- $\alpha$ forest. J. Cosmol. Astroparticle Phys. DOI: $10.1088 / 1475-7516 / 2006 / 06 / 019$

Kurth, R., 1972. Dimensional Analysis and Group Theory in Astrophysics. Pergamon Press, Oxford, ISBN: 0080166164

Petty, G.W., 2001. Automated computation and consistency checking of physical dimensions and units in scientific programs. Software Practice Experience, 31: 1067-1076. DOI: 10.1002/spe.401
Planck, M., 1959. The Theory of Heat Radiation. Dover Publications, New York. ISBN: 1114813141 (translation from German Ed. 1906)

Rodriguez-Fernandez, J. L., 1999. Ockham's Razor. Endeavour, 23: 121-125. DOI: $10.1016 / \mathrm{S} 0160-9327(99) 01199-0$

Sivaram, C., 1982. Cosmological and quantum constraint on particle masses. Am. J. Phys., 50: 279. DOI: $10.1119 / 1.12870$

Valev, D., 2008. Neutrino and graviton mass estimations by a phenomenological approach. Aerospace Res. Bulg., 22: 68-82.

Valev, D., 2009. Determination of total mechanical energy of the universe within the framework of Newtonian mechanics. Preprint: arXiv: 0909.2726

Valev, D., 2013. Three fundamental masses derived by dimensional analysis. Am. J. Space Sci., 1: 145-149. DOI: 10.3844/ajssp.2013.145.149

Weinberg, S., 1972. Gravitation and Cosmology. Wiley, New York. ISBN: 0471925675

Woodward, J.F., R.J. Crowley and W. Yourgrau, 1975. Mach's principle and the rest mass of the graviton. Phys. Rev. D, 11: 1371-1374.

DOI: 10.1103/PhysRevD.11.1371 